\title{
Does Type of Disability Matter to Public Health Policy and Practice?
}

\author{
Amanda Reichard, Michelle Stransky, Kimberly Phillips, \\ Charles Drum, and Monica McClain \\ Institute on Disability, University of New Hampshire
}

\begin{abstract}
Background: Surveillance has been insufficient to inform and evaluate public health practices for people with disabilities. No studies have investigated whether there is statistical justification for subdividing the large, heterogeneous group of people with disabilities into subpopulations, for surveillance.

Methods: Pooled data from the Medical Expenditures Panel Survey $(2004-2010$, unweighted n=132,198) included the following disability types: physical, cognitive, visual, hearing loss, and multiple disabilities. We examined differences among the disability subgroups and the no disability group on receipt of flu shot, dental exam, and timely care; insurance status; usual source of care (USOC); mental and physical health; and multiple chronic conditions (MCC).

Results: The disability subgroups were sociodemographically heterogeneous and differed from each other and the no disability group on health status (mental, physical, and MCC) and healthcare outcomes (flu shot, dental exam, timely receipt of care, USOC, insurance status).

Conclusion: Findings demonstrate that disability subgroups differ in the magnitude of the disparities they experience compared to each other and to people without disabilities. Disability subgroups should be examined separately for public health measures to enable effective tailoring of public health policies and programs to better meet the needs for all people.
\end{abstract}

(c) 2015 Californian Journal of Health Promotion. All rights reserved.

Keywords: disability, public health surveillance, health status, health outcomes

\section{Introduction}

Recent efforts have highlighted the poor health of people with disabilities (Horner-Johnson, Dobbertin, Lee, \& Andresen, 2013; 2014; Reichard, Stolzle, \& Fox, 2011); however few public health efforts have been directed to or purposefully included this population. Nearly $19 \%$ of U.S. adults have a disability (Brault, 2012), defined as people with substantial functional limitations in physical function, cognitive function, vision, or hearing. While recent support from federal agencies (Centers for Disease Control and Prevention, 2011; U.S. Department of Health and Human Services, 2002, 2005) has enabled a substantial increase in the number of intervention studies aimed at improving health outcomes for individuals with disabilities (Naaldenberg, Kuijken, van Dooren, \& van Schrojenstein Lantman de Valk, 2013; Seekins, Drum, Kimpton, \& al., 2010), a sufficient concomitant surveillance effort has not been undertaken to inform which interventions are needed to address the disparities experienced among these subgroups and to evaluate the effects of interventions and other public health practices on this population. Instead, surveillance related to this population has continued to focus on estimating prevalence of birth defects, developmental disabilities, neurological diseases (McGeehin, Qualters, \& Niskar, 2004) and spinal cord and traumatic brain injury (Adams, Krahn, Horner-Johnson, \& Leman, 2009; Coronado et al., 2011).

Nsubuga et al. stated (2006), "Public health surveillance provides the scientific and factual database essential to informed decision making and appropriate public health action (p. 2)." Purposeful and comprehensive expansion of surveillance efforts specific to people with disabilities is required to best address the health 
disparities that individuals with disabilities experience (Krahn, Walker, \& Correa DeAraujo, 2015). These efforts are necessary not only to enumerate individuals with disabilities but also to guide public health action inclusive of people with disabilities. Additionally, such work is necessary to assess the extent of progress of public health programs and interventions in mediating the effects of social factors (e.g., poverty, discrimination) on health outcomes, quality of life, and function among people with disabilities (Adams et al., 2009).

Research on disparities among people with disabilities has typically compared people with disabilities to those without them. Some intervention studies have subdivided the disability population (Havercamp, Scandlin, \& Roth, 2004; Jones \& Lollar, 2008; Kancherla, Van Naarden Braun, \& Yeargin-Allsopp, 2013; Rimmer, Wang, Pellegrini, Lullo, \& Gerber, 2013) because ample conceptual and experiential evidence strongly suggests important differences among persons with disabilities based on disability type (physical, cognitive, developmental, sensory impairments, mental health) (Anderson et al., 2013; Reichard \& Stolzle, 2011; Reichard et al., 2011; Shireman, Reichard, \& Hunt, 2013). However, only a handful of studies have compared outcomes among subgroups of the disability populations. No studies have investigated whether there is statistical justification for subdividing the larger, heterogeneous group of people with disabilities into subpopulations for surveillance research, to determine whether specific disability types vary in ways that necessitate unique practical response to appropriately address the barriers and disparities experienced by these subgroups.

\section{The Current Study}

This study was one of the first to offer a model to purposefully increase and improve surveillance on individuals with disabilities. Specifically, this study compared disability subgroups and the no disability group on receipt of flu shot, dental exam, and timely care; mental and physical health status; multiple chronic conditions (MCC); usual source of care (USOC); and, insurance status; to determine whether future surveillance efforts should consider monitoring outcomes by disability type. The objective was to confirm or refute, through statistical testing, conceptual and experiential evidence of differences between and among people with different types of disabilities that are relevant to public health surveillance.

\section{Methods}

\section{Study Design}

We analyzed pooled data from the Medical Expenditures Panel Survey (MEPS), 2004-2010. The MEPS is frequently used for health services research (Agency for Healthcare Research and Quality, 2009). MEPS has become the premier dataset for examining health care access disparities in vulnerable populations with research conducted among older adults, the uninsured, rural Americans, children with special health care needs, and women (Alegría et al., 2006; Shi, 2000). All variables used for this study have been included in the MEPS since at least 2000.

\section{Participants}

We included all working age adults (18-64) who had values for all study measures in the analyses $(n=132,198)$. The MEPS includes a subsample of respondents from the National Health Interview Survey (NHIS), and is nationally representative of the civilian noninstitutionalized population in the United States. The NHIS uses state-based stratification to sample respondents, and it oversamples racial and ethnic minorities (Parsons, Mariarity, Jonas, \& al., 2014). Self-reported information is collected on all people in selected households five times over a two-year span; all data in this study are from the household component of the MEPS.

\section{Measures}

Outcome Variables. We examined a total of eight health and healthcare items, including five healthcare and health status measures and three healthcare use measures. The healthcare and health status measures were (1) self-reported physical health, (2) self-reported mental health, (3) multiple chronic conditions (MCC), (4) 
having health insurance, and (5) untimely receipt of care. The health care use measures were: (6) receipt of flu vaccine, (7) receipt of dental exam, and (8) having a usual source of care (USOC). Self-reported physical health and self-reported mental health were collapsed into excellent/very good/good (1) versus fair/poor (0), as is commonly done (e.g., Horner-Johnson, Dobbertin, Lee, \& Andresen, 2013; Reichard, Gulley, Rasch, \& Chan, 2015). Having MCC was defined as having any two or more of the following priority health conditions: arthritis, asthma, cardiovascular disease, diabetes, emphysema, high blood pressure, or stroke $(0=$ No; $1=$ Yes). Responses to receipt of dental examination and receipt of flu vaccine within the last year were measured dichotomously $(0=$ No; $1=$ Yes). Anyone who indicated that they had a USOC (other than the ER) was considered to having a USOC (1=Yes); those who reported using the ER or responded 'no' to this question were considered to not have a USOC $(0=\mathrm{No})$, per the Healthy People 2020 definition of ongoing sources of care. People were coded as having insurance if they had any private insurance and public insurance only during the year $(1=$ Yes); those who were not insured for the entire year were considered uninsured $(0=$ No $)$. Finally, untimely receipt of care measure pertained to self-reported delaying or not receiving necessary medical services, dental services, or prescription medications (1=Yes); people who never reported having had to delay or not receive necessary care were coded as such $(0=\mathrm{No})$. We selected these measures because they are all commonly used in public health surveillance in the U.S. (U.S. Department of Health \& Human Services, 2010a).

Predictor Variables. The predictor of interest was disability subgroup. We classified the analytic sample into one of six mutually exclusive groups (five disability and one nondisabled). A person was considered to have a cognitive limitation if they answered 'yes' at two points in time to: "experiencing confusion or memory loss, having problems making decisions, or requiring supervision for their own safety" (unweighted $\mathrm{n}=527$ ) (Agency for Healthcare Research and Quality, 2009, pp. C40). Physical limitations were identified based on at least one affirmative report of "difficulties walking, climbing stairs, grasping objects, reaching overhead, lifting, bending or stooping, or standing for long periods of time” (unweighted $n=12,526$ ) (Agency for Healthcare Research and Quality, 2009, pp. C-38). Visual impairments were defined as being "impaired near, but not far", "impaired both near and far", or "blind," even when wearing contacts or glasses (if used) (unweighted $n=814$ ). The hearing loss group included people who reported having a moderate or major impairment, or being deaf (unweighted $n=386$ ). Anyone who responded "yes" to at least two of these limitations was considered to have multiple impairments (unweighted $n=2,563$ ). People not reporting any impairments or limitations were classified as having no disabilities (unweighted $n=121,622$ ); these people were included in the bivariate analyses but not the Wald testing. Recent research has shown that these differences exist in health and healthcare outcomes for these subgroups (Dobbertin, Horner-Johnson, Lee, \& Andresen, 2014; Horner-Johnson et al., 2014) and between people with disabilities and people without disabilities (McDermott, Moran, Platt, \& Dasari, 2006; Reichard, Stolzle, \& Fox, 2011).

Demographic Covariates. Covariates included age (continuous, 18-64), sex (male/female), and race/ethnicity (non-Hispanic white, nonHispanic black, non-Hispanic other, and Hispanic). They also included education (<high school, high school, > high school), income (poor/near poor $(>125 \%$ of the federal poverty line, other)), married (yes/no), region (Northeast, Midwest, South, West), and metropolitan statistical area (MSA; yes/no). We also included need for assistance with instrumental activities of daily living (IADLs) and activities of daily living (ADLs) because this characteristic has been shown to predict need for and use of health care services (Gulley, Rasch, \& Chan, 2011). Survey respondents who reported "receiv[ing] help or supervision with personal care such as bathing, dressing, or getting around the house" (ADLs) or "receiv[ing] help or supervision using the telephone, paying bills, taking medications, preparing light meals, doing laundry, or going shopping” (IADLs) (Agency for Healthcare 
Research and Quality, 2009; C-37-38) were coded as having these assistance needs.

\section{Analyses}

We conducted descriptive analyses, t-tests and pairwise chi-square analyses to compare sociodemographic factors, health status, and healthcare use outcomes across the five disability subgroups (physical limitations, cognitive limitations, visual impairments, deaf/hard of hearing, and multiple limitations) and the no disability group. Next, we predicted each of the eight outcomes using omnibus and pairwise logistic regression and Wald tests among the disability subgroups; people without disabilities were excluded from these analyses. For each outcome, an omnibus Wald test was conducted to ascertain whether the group of covariates varied across the five subpopulations, when all of them were considered jointly. In addition, to determine the specific subpopulations that should be modeled separately, Wald tests and logistic regression analyses were conducted for pairwise combinations of the five subpopulations, for each of the health outcomes. The pairwise Wald test was used to compare the differences in the outcomes of interest for each pair of disability groups, though their interactions with each sociodemographic characteristic. The Wald test was used to examine USOC, untimely receipt of care, health insurance, and MCC were included as covariates in the analyses when they were not the outcome of interest. This method has previously been used to investigate the unique impact of sets of predictors on an outcome (Korn \& Graubard, 1990; Mulvaney-Day,
Alegría, \& Sribney, 2007). The logistic regression results are neither shown nor discussed in this paper. We believe that those results deserve a more extensive, in-depth and thorough treatment than they can be given here due to our interest in the Wald test results and the diversity in our outcomes. The results were substantively similar when reduced sets of predictors were used, thus multi-collinearity was not a concern. Sample weights and design variables were used in all analyses to adjust for the complex sample design of the MEPS. All analyses were conducted using Stata MP/13 (College Station, TX).

\section{Results}

Our weighted sample of working-age adults (1864 ) included $9.8 \%$ with physical limitations, $1.7 \%$ with multiple impairments, and less than $1 \%$ each reporting cognitive limitations, hearing impairments, and visual impairments; the remainder did not report a limitation. First, we summarized the statistically significant bivariate differences in sociodemographic and health and healthcare outcomes among the five disability groups. (We do not highlight the differences between individuals within each disability subgroup and those without disabilities because these differences are already well-established and is not the specific focus of this work; however, the findings for the no disability group are included in Table 1 , for reference). 
Table 1.

Sociodemographic and Health Characteristics, by Disability Subtype

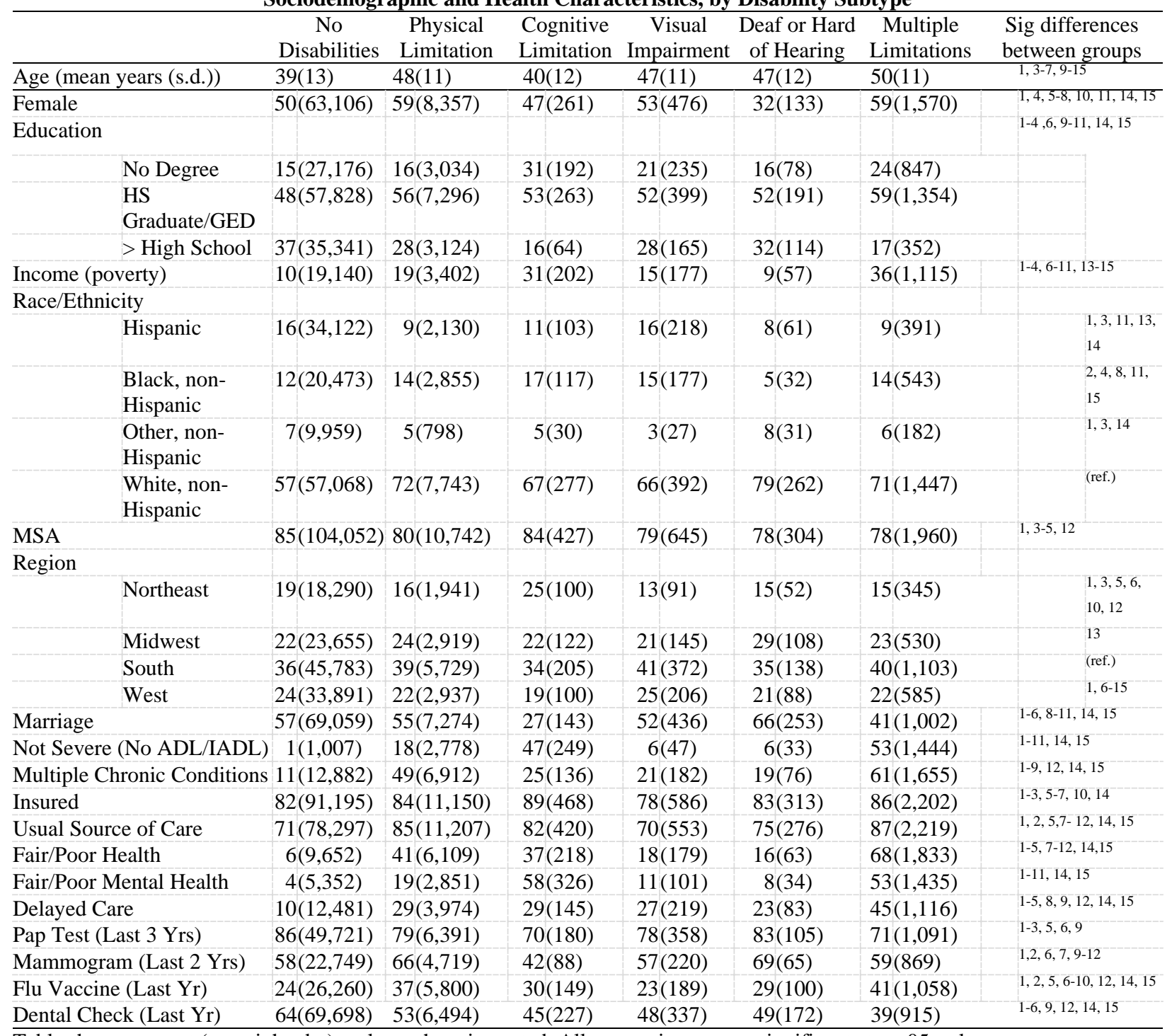

Table shows percent (unweighted $\mathrm{n}$ ), unless otherwise noted. All comparisons were significant at $\mathrm{p}<.05$ or lower.

(1) No disability vs. physical limitation, (2) no disability vs. cognitive limitation, (3) no disability vs. visual impairment, (4) no disability vs. deaf/hard of hearing, (5) no disability vs. multiple limitations, (6) physical limitation vs. cognitive limitation, (7) physical limitation vs. visual impairment, (8) physical limitation vs. deaf/hard of hearing, (9) physical limitation vs. multiple limitations, (10) cognitive limitations vs. visual impairment, (11) cognitive limitation vs. deaf/hard of hearing, (12) cognitive limitation vs. multiple limitation, (13) visual impairment vs. deaf/hard of hearing, (14) visual impairment vs. multiple limitations, (15) deaf/hard of hearing vs. multiple limitations.

\section{Characteristics of Disability Groups}

Bivariate analyses revealed substantial differences in the sociodemographic profiles of the disability subgroups and no disability group (Table 1). Additionally, health and healthcare outcomes differed between the disability subgroups and the no disability group such that the proportion of people in each of the disability subgroups who reported untimely receipt of care, MCC, and fair/poor health was double the proportion of people with no disabilities.

Physical Limitations. Compared to people with other types of single disabilities (cognitive limitations, hearing limitations, vision 
limitations), people with physical limitations were more likely to be female (58.5\%), to have a usual source of care (85\%), and to have had a flu vaccine in the past year (37\%). Nearly half (49\%) of people with physical limitations experience MCC, which was about double the prevalence among the next highest group (25\% among people with cognitive limitations) and nearly five times higher than people without limitations (11\%). People with physical limitations also comprised one of two groups (along with people with cognitive limitations) most likely to self-report health status as fair or poor $(41 \%)$, and almost one-third (29\%) of this group reported having untimely receipt of care.

Cognitive Limitations. Compared to people with other types of single disabilities and people with no limitations, people with cognitive limitations were least likely to have a high school education (69\%). They were also most likely to be poor (31\%) and least likely to be married (27\%). Insurance coverage (88.8\%) was high among this group, and the prevalence of untimely receipt of care (29\%) was nearly equivalent to that of people with physical limitations. People with cognitive limitations were as likely as people with physical limitations to report fair or poor health (37\%), and they were more likely than any other group (58\%) to say that their mental health is fair or poor. Moreover, people with cognitive limitations were significantly more likely than any other group with single disabilities or no disabilities to require assistance with IADL/ADLs (47\%).

Visual Impairments. The prevalence of Hispanic race/ethnicity among people with visual impairment (15.9\%) was equivalent to that among the individuals with no limitations (16\%); both of these are higher than the prevalence among people with any other type of single disability. People with visual impairments are less likely to be insured than any other group (78\%), except people with hearing impairment. People with vision impairments were least likely (along with hearing loss group) of other single disability groups to need assistance with IADLs/ADLs (6\%). Although five to six percent prevalence represents a significant need compared to people with no limitations (1\%), it was also substantially lower than the need among people with physical limitations (18\%) and people with cognitive limitations (47\%). Similarly, people with vision impairments were less likely than people with physical, cognitive, and multiple limitations to report fair or poor physical health (18\%) and fair or poor mental health (11\%). However, they were significantly more likely to report these outcomes compared to individuals with no disability (6\% and $4 \%$, respectively).

Hearing Loss. Compared to people with other types of single disabilities and people with no limitations, people with hearing impairment were more likely to be male (58\%), less likely to be Hispanic (8\%) or Black (5\%), and more likely to be married (66\%). As previously described (see Visual Impairments), people with hearing loss were less likely than people with most other types of single disabilities to require assistance with IADLs/ADLs (6\%), and they were less likely to report their physical and mental health status as fair or poor $(16 \%, 8 \%$, respectively). Similar to those with vision impairments (6\%), individuals with hearing loss were least likely among the disability groups to require assistance with ADLs/IADLs, but more likely than those with no disability $(0.8 \%)$ to require this help. The prevalence of fair or poor physical health among people with hearing loss was much lower than other disability groups, but substantially higher than the reported rates among people with no limitations for physical health and mental health, respectively (6\% and $4 \%$, respectively).

Multiple Limitations. In the current study, 98\% of people with multiple disabilities had a physical limitation and at least one other disability type. Perhaps the most striking characteristics of people with multiple disabilities compared to people with a single type of disability and people with no limitations were the overwhelming prevalence of MCC (61\%). Individuals with multiple limitations were more likely than people with one or no limitations to report a USOC (87\%) and to have health insurance (61\%). Nonetheless, they were also most likely to report untimely receipt of 
medical care (45\%); the groups second most likely to report delaying needed care were people with either physical or cognitive limitations alone, with 29\%). Low income may account for some of the delayed care, as people with multiple limitations were more likely than other groups except cognitive limitations to be poor (36\%).

People with multiple limitations also reported a significantly higher prevalence (52\%) of assistance needs for IADLs/ADLs than any other group besides people with cognitive limitations (47\%). Moreover, self-reports of overall health were rated fair or poor by more than two-thirds (68\%) of this group, which was much higher than any other group (next closest was people with physical limitations (41\%)). Individuals with multiple limitations also rated their mental health as fair or poor more often (53\%) than any other group except people with cognitive limitations (58\%).

\section{Omnibus Tests Controlling for Sociodemographic Factors}

The omnibus test for each of the health and healthcare outcomes resulted in F-ratios that were significant at $\mathrm{p}<0.001$. This suggests that, for each of the health outcomes, the explanatory power of the full set of variables, together, varied across the disability subpopulations. Therefore, the disability subgroups should be modeled separately rather than as a single crossdisability group.

Tests to determine which disability subgroups should be examined independently, for each outcome (the last column in Table 2) suggest that the disability subpopulations, overall, have sufficiently unique attributes to statistically justify analyzing them separately. For both health insurance status and MCC, pairwise comparisons were statistically significant for all subgroups except cognitive limitations versus hearing loss. Physical health status pairwise group comparisons were significant for all pairs except visual limitations versus hearing loss; mental health status comparisons were significant for all pairs except visual limitations versus hearing loss and cognitive limitations versus multiple limitations (though this pair neared significance, $\mathrm{p}<0.05$ ).
Table 2.

Results of Wald Tests for Omnibus and Pairwise Combinations of Disability

Sub-populations, by Health Care Status/Healthcare use Measure

\begin{tabular}{|c|c|c|}
\hline \\
\hline Measures & $\begin{array}{c}\text { Omnibus } \\
\text { (Wald } \\
\text { value) }\end{array}$ & $\begin{array}{l}\text { Sig. Pairwise } \\
\text { combinations }\end{array}$ \\
\hline Flu Shot & $2.26^{*}$ & $1,4,5,6$ \\
\hline Dental Exam & $3.77^{*}$ & 4 \\
\hline Delayed/Not Received & & \\
\hline Needed Care & $6.47^{*}$ & $1,4,7,9,10$ \\
\hline Usual Source of Care & $3.09^{*}$ & $1,2,4,9$ \\
\hline Insured & $5.53^{*}$ & $1-5,7-10$ \\
\hline Physical Health Status & $6.39^{*}$ & $1-7,9,10$ \\
\hline Mental Health Status & $13.33^{*}$ & $1,3-6,9,10$ \\
\hline $\begin{array}{l}\text { Multiple Chronic } \\
\text { Conditions }\end{array}$ & $6.86^{*}$ & $1-5,7-10$ \\
\hline$(*)=p<.001$ & & \\
\hline $\begin{array}{l}\text { (1) physical limitation } \\
\text { physical limitation vs. } \\
\text { limitation vs. deaf/hard } \\
\text { limitation vs. multiple } \\
\text { limitations vs. visual in } \\
\text { limitation vs. deaf/hard } \\
\text { limitation vs. multiple } \\
\text { impairment vs. deaf/ha } \\
\text { impairment vs. multipl } \\
\text { hearing vs. multiple lin }\end{array}$ & $\begin{array}{l}\text { gnitive limi } \\
\text { l impairmen } \\
\text { earing, (4) p } \\
\text { tions, (5) cc } \\
\text { ment, (6) co } \\
\text { earing, (7) c } \\
\text { tion, (8) vis } \\
\text { hearing, (9) } \\
\text { tations, (10) } \\
\text { ons }\end{array}$ & $\begin{array}{l}\text { ation, (2) } \\
\text { (3) physical } \\
\text { yysical } \\
\text { gnitive } \\
\text { nitive } \\
\text { gnitive } \\
\text { al } \\
\text { visual } \\
\text { deaf/hard of }\end{array}$ \\
\hline
\end{tabular}

Assessed from the point of view of the pairwise combinations, each pair of disability subpopulations revealed statistical significance for at least three of the health and healthcare outcomes. For example, subgroup differences were found for cognitive limitations and visual impairments for receipt of flu shot, health insurance, physical health status, mental health status, and multiple chronic conditions.

\section{Discussion}

Epidemiologic research contrasting health and healthcare outcomes for individuals with and without disabilities provides us with valuable information necessary to inform public health policy, practice, and interventions. To date, surveillance efforts have typically focused on measuring the prevalence of certain disabilities and have not consistently provided the empirical data necessary for evidence-based decisions and relevant public health actions, including determining the need for and evaluating the effect of interventions. Monitoring the 
prevalence and distribution of poor health and healthcare outcomes among individuals with disabilities is critical to identifying those who fare the worst to inform changes in relevant policies and practices (Drum, Krahn, Culley, \& Hammond, 2005; Krahn \& Campbell, 2011; Krahn, Hammond, \& Turner, 2006). Although our findings did not show that all health and healthcare outcomes differ by disability subgroup, the evidence is sufficient across outcomes to support the use of subgroup analyses. These findings support the framework that the best way to examine people with disabilities is through a disability subgroup framework, not a single, cross-disability group to adequately assess where disparities exist and where to most effectively target intervention efforts.

These results showed that regardless of the disability type, health and healthcare outcomes differ among the disability subgroups. This confirms what we know about the unique needs and distinct barriers to care experienced by people with different types of disabilities (Naaldenberg et al., 2013; Reichard et al., 2015; Seekins et al., 2010). The findings also confirmed the conceptual and experiential evidence in support of examining disability subgroups, separately, as documented by others (Dobbertin et al., 2014; Drainoni et al., 2006; Willi Horner-Johnson \& Dobbertin, 2014; 2013; McDermott, Moran, Platt, \& Dasari, 2006; Reichard \& Fox, 2013; Reichard \& Stolzle, 2011; Reichard, Stolzle, Sella, \& Shireman, 2012). These results further demonstrated the need for formal and comprehensive surveillance of disability subpopulations that can appropriately inform interventions to meet the unique needs of each these subgroups.

As numerous others have identified, there was a great need to establish better definitions not only of disabilities, broadly, but also for the specific disability subgroups. Including a set of standardized identifiers will help researchers, program designers, and policymakers to a) better monitor demographic change, b) better identify health disparities within subgroups, c) improve the precision in monitoring health status among subgroups, and d) strengthen capacity to target local, state and national interventions. Such an approach has been advocated by the Institute of Medicine regarding the importance of standardized data collection of race, ethnicity and language to allow for quality improvements designed to identify and address disparities among such subgroups (Institute of Medicine, 2003, 2009).

Although substantially downstream from our findings, the implications of this work may appear to support the continued implementation of specialized public health programs that target specific categories of disability and diagnoses, such as a diabetes management program specifically for people with physical limitations. However, such programs can be costly and difficult to sustain. Moreover, creating separate disability-specific programs contradicts our obligation to include people with disabilities to the greatest extent possible in mainstream public health promotion and activities ("Americans with Disabilities Act of 1990," 1990; U.S. Department of Health \& Human Services, 2010c). The findings of this study demonstrate that a thorough understanding of the unique attributes of each disability subgroup is essential for ensuring universal access.

\section{Limitations}

Limitations inherent to the MEPS data may have an effect on the findings of this study. First, the MEPS relies on self-reports that can result in errors from poor recall or understanding. The study sample contains a proportion of individuals who may have cognitive limitations or related disabilities that can affect their response accuracy. In addition, bias may be increased through proxy response if proxies have inaccurate information relevant to the questions. Second, the questions available on the MEPS for identifying individuals with "cognitive limitations" are broad and likely cause the inclusion of disabilities ranging from intellectual disability to dementia. Third, the small sample sizes of some of the disability subpopulations likely reduced the statistical power of some analyses. However, many of the results approached statistical significance, warranting further research with larger sample sizes for these groups (cognitive limitations, 
visual impairments, hearing loss), especially given what is known about the differences in the resources available these populations, the barriers they face, and their health services utilization patterns (Drum, Krahn, Culley, \& Hammond, 2005; Harrington \& Kang, 2008; Kang \& Harrington, 2008; Krahn, Fox, Campbell, Ramon, \& Jesien, 2010; U.S. Department of Health and Human Services, 2002; Ward, Nichols, \& Freedman, 2010). Finally, the sample included only the noninstitutionalized population; thus it did not capture the experiences of the many individuals with disabilities living in institutional settings
(Agency for Healthcare Research and Quality, 2009).

\section{Conclusion}

In summary, purposefully and thoroughly expanding surveillance of individuals with disabilities can very possibly improve the ability of public health experts to meet the needs of this population. In particular, conducting surveillance research separately on disability subgroups, the goal is to identify and address the needs of all people, including those in disability subgroups, to the greatest extent possible in mainstream public health programs and policies.

\section{References}

Adams, E., Krahn, G., Horner-Johnson, W., \& Leman, R. (2009). Fundamentals of Disability Epidemiology. In C. E. Drum, G. Krahn, \& H. Bersani, Jr. (Eds.), Disability and Publich Health (pp. 105-124). Washington, DC: American Association on Intellectual and Developmental Disabilities.

Agency for Healthcare Research and Quality. (2009). MEPS HC-113: 2007 Full Year Consolidated Data File. In A. Center for Financing, and Cost Trends (Ed.). Rockville, MD.

Alegría, M., Cao, Z., McGuire, T. G., Ojeda, V. D., Sribney, B., Woo, M., \& Takeuchi, D. (2006). Health insurance coverage for vulnerable populations: contrasting Asian Americans and Latinos in the United States. INQUIRY: The Journal of Health Care Organization, Provision, and Financing, 43(3), 231-254.

Americans with Disabilities Act of 1990, Pub. L. § No. 101-336 (U. S. Department of Justice 1990).

Anderson, L. L., Humphries, K., McDermott, S., Marks, B., Sisarak, J., \& Larson, S. (2013). The state of the science of health and wellness for adults with intellectual and developmental disabilities. Intellect Dev Disabil, 51(5), 385-398. doi: 10.1352/1934-9556-51.5.385

Brault, M. (2012). Americans With Disabilities: 2010. Washington, DC.

Centers for Disease Control and Prevention. (2011). CDC health disparities and inequalities report United States, 2011 MMWR. Mobility and Mortality Weekly Report (Vol. Supplement, Vo. 60, pp. 116).

Coronado, V. G., Xu, L., Basavaraju, S. V., McGuire, L. C., Wald, M. M., Faul, M. D., . . Hemphill, J. D. (2011). Surveillance for traumatic brain injury-related deaths: United States, 1997-2007: US Department of Health and Human Services, Centers for Disease Control and Prevention Atlanta.

Dobbertin, K., Horner-Johnson, W., Lee, J. C., \& Andresen, E. M. (2014). Subgroup differences in having a usual source of health care among working-age adults with and without disabilities. Disability and health journal.

Drainoni, M.-L., Lee-Hood, E., Tobias, C., Bachman, S. S., Andrew, J., \& Maisels, L. (2006). Crossdisability experiences of barriers to health-care access consumer perspectives. Journal of Disability Policy Studies, 17(2), 101-115.

Drum, C. E., Krahn, G., Culley, C., \& Hammond, L. (2005). Recognizing and responding to the health disparities of people with disabilities. California Journal of Health Promotion, 3(3), 29-42.

Gulley, S. P., Rasch, E. K., \& Chan, L. (2011). The complex web of health: relationships among chronic conditions, disability, and health services. Public Health Reports, 126(4), 495-507. 
Harrington, C., \& Kang, T. (2008). Disparities in service utilization and expenditures for individuals with developmental disabilities. Disability and Health Journal, 1(4), 184-195. doi: 10.1016/j.dhjo.2008.05.004

Havercamp, S. M., Scandlin, D., \& Roth, M. (2004). Health disparities among adults with developmental disabilities, adults with other disabilities, and adults not reporting disability in North Carolina. Public Health Reports, 119(4), 418-426.

Horner-Johnson, W., \& Dobbertin, K. (2014). Usual Source of Care and Unmet Health Care Needs: Interaction of Disability With Race and Ethnicity. Medical care, 52, S40-S50.

Horner-Johnson, W., Dobbertin, K., Lee, J., \& Andresen, E. (2013). Disparities in chronic conditions and health status by type of disability. Disability and Health Journal, 6(4), 280-286.

Horner-Johnson, W., Dobbertin, K., Lee, J. C., \& Andresen, E. M. (2014). Disparities in Health Care Access and Receipt of Preventive Services by Disability Type: Analysis of the Medical Expenditure Panel Survey. Health services research.

Institute of Medicine. (2003). The future of the public's health in the 21st century. Washington, D.C.: National Academies Press.

Institute of Medicine. (2009). Race, Ethnicity, and Language Data: Standardization for Health Care Quality Improvement. Washington, DC: The National Academies Press.

Jones, S. E., \& Lollar, D. J. (2008). Relationship between physical disabilities or long-term health problems and health risk behaviors or conditions among US high school students. Journal of School Health, 78(5), 252-257.

Kancherla, V., Van Naarden Braun, K., \& Yeargin-Allsopp, M. (2013). Dental care among young adults with intellectual disability. Res Dev Disabil, 34(5), 1630-1641. doi: 10.1016/j.ridd.2013.02.006

Kang, T., \& Harrington, C. (2008). Variation in types of service use and expenditures for individuals with developmental disabilities. Disability and Health Journal, 1(1), 30-41. doi: 10.1016/j.dhjo.2007.11.008

Korn, E. L., \& Graubard, B. I. (1990). Simultaneous Testing of Regression Coefficients with Complex Survey Data: Use of Bonferroni t Statistics. The American Statistician, 44(4), 270-276.

Krahn, G., Fox, M. H., Campbell, V. A., Ramon, I., \& Jesien, G. (2010). Developing a health surveillance system for people with intellectual disabilities in the United States. Journal of Policy and Practice in Intellectual Disabilities, 7(3), 155-166.

Krahn, G., \& Campbell, V. A. (2011). Evolving views of disability and public health: the roles of advocacy and public health. Disability and Health Journal, 4(1), 12-18.

Krahn, G., Walker, D. K., \& Correa DeAraujo, R. (2015). Persons With Disabilities as an Unrecognized Health Disparity Population. American Journal of Public Health, 105(S2), S198-S206.

Krahn, G., Hammond, L., \& Turner, A. (2006). A cascade of disparities: Health and health care access for people with intellectual disabilities. Mental Retardation and Developmental Disabilities Research Reviews, 12(1), 70-82.

McDermott, S., Moran, R., Platt, T., \& Dasari, S. (2006). Variation in health conditions among groups of adults with disabilities in primary care. Journal of Community Health, 31(3), 147-159.

McGeehin, M. A., Qualters, J. R., \& Niskar, A. S. (2004). National Envioronmental Public Health Tracking Program: Bridging the Information Gap. Environmental Health Perspectives, 112(14), 1409-1413.

Mulvaney-Day, N. E., Alegría, M., \& Sribney, W. (2007). Social cohesion, social support, and health among Latinos in the United States. Social Science \& Medicine, 64(2), 477-495.

Naaldenberg, J., Kuijken, N., van Dooren, K., \& van Schrojenstein Lantman de Valk, H. (2013). Topics, methods and challenges in health promotion for people with intellectual disabilities: A structured review of literature. Research in Developmental Disabilities, 34(12), 4534-4545.

Nsubuga, P., White, M. E., Thacker, S. B., \& et al. (2006). Public Health Surveillance: A Tool for Targeting and Monitoring Interventions. In D. T. Jamison, J. G. Breman, A. R. Measham, \& et al. (Eds.), Disease Control Priorities in Developing Countries (2nd ed.). Washington, DC: World Bank. 
Parsons, V. L., Mariarity, C., Jonas, K., \& al., E. (2014). Design and Estimation for the National Health Interview Survey, 2006-2015. Vital and Health Statistics. Series 2: Data Evaluation and Methods Research, 2(165).

Reichard, A., \& Fox, M. H. (2013). Using population-based data to examine preventive services by disability type among dually eligible (Medicare/Medicaid) adults Disability and Health Journal, 6(2), 75-86.

Reichard, A., Gulley, S. P., Rasch, E. K., \& Chan, L. (2015). Diagnosis isn't enough: Understanding the connections between high health care utilization, chronic conditions and disabilities among US working age adults. Disability and Health Journal.

Reichard, A., Saunders, M., Saunders, R., Donnelly, J., Lauer, E., Sullivan, D., \& Ptomey, L. (2015). A Comparison of Two Weight Management Programs for Adults with Mobility Impairments. Disability and Health Journal, 8(1), 61-69.

Reichard, A., \& Stolzle, H. (2011). Diabetes Among Adults with Cognitive Limitations Compared to Individuals with No Cognitive Disabilities. Disability and Health Journal, 49(3), 141-154.

Reichard, A., Stolzle, H., \& Fox, M. H. (2011). Health disparities among adults with physical disabilities or cognitive limitations compared to individuals with no disabilities in the United States. Disability and Health Journal, 4(2), 59-67.

Reichard, A., Stolzle, H., Sella, A. C., \& Shireman, T. I. (2012). Quality of diabetes care for adults with physical disabilities. Disability and Health Journal, 5(1), 34-40.

Rimmer, J., Vanderbom, K. A., Bandini, L. G., Drum, C., Luken, K., Suzarez-Balcazar, Y., \& Graham, I. (2014). GRAIDs: a framework for closing the gap in the availability of health promotion programs and interventions for people with disabilities. Implementation Science, 9(100), 1-9.

Rimmer, J., Wang, E., Pellegrini, C. A., Lullo, C., \& Gerber, B. S. (2013). Telehealth weight management intervention for adults with physical disabilities: a randomized controlled trial. American Journal of Physical Medicine and Rehabilitation, 92(12), 1084-1094.

Seekins, T., Drum, C., Kimpton, T., \& al., E. (2010). Community-Based Health Promotion Interventions for People with Disabilities. Portland, OR: Rehabilitation Research and Training Center on Health and Wellness for Persons with Long Term Disabilities.

Shi, L. (2000). Vulnerable Populations and Health Insurance. Medical Care Research and Review, 57(1), 110-134.

Shireman, T. I., Reichard, A., \& Hunt, S. L. (2013). Learning your ABDs: variation in health care utilization across Kansas Medicaid disability groups. Disability and Health Journal, 6(3), 220226.

U.S. Department of Health \& Human Services. (2010a). Healthy People 2020: An Opportunity to Address Societal Determinants of Health in the United States.

U.S. Department of Health \& Human Services. (2010c). Healthy People 2020: Disability and Health.

U.S. Department of Health and Human Services. (2002). Closing the Gap: A National Blueprint to Improve the Health of Persons with Mental Retardation: Report of the Surgeon General's Conference on Health Disparities and Mental Retardation. Rockville, MD: Office of the U.S. Surgeon General.

U.S. Department of Health and Human Services. (2005). The Surgeon General's call to action to improve health and wellness of people with disabilities. In U. S. D. o. H. a. H. Services (Ed.): U.S. Government.

Ward, R. L., Nichols, A. D., \& Freedman, R. I. (2010). Uncovering Health Care Inequalities among Adults with Intellectual and Developmental Disabilities. Health \& Social Work, 35(4), 280-290. 


\author{
Author Information: \\ *Amanda Reichard, PhD \\ Associate Director of Disability and Health Policy \\ Institute on Disability \\ University of New Hampshire \\ 10 West Edge Dr., Suite 101 \\ Durham, NH, 03824 \\ Amanda.Reichard@unh.edu \\ 603-862-5266 \\ * corresponding author
}

